\title{
SEMILATTICES WITH A TRANSITIVE AUTOMORPHISM GROUP
}

\author{
F. PASTIJN \\ (Received 14 March 1979) \\ Communicated by T. E. Hall
}

\begin{abstract}
If $L$ is any semilattice, let $T_{L}$ denote the Munn semigroup of $L$, and Aut $(L)$ the automorphism group of $L$.

We show that every semilattice $L$ can be isomorphically embedded as a convex subsemilattice in a semilattice $L^{\prime}$ which has a transitive automorphism group in such a way that

(i) every partial isomorphism $\alpha$ of $L$ can be extended to an automorphism $\bar{\alpha}$ of $L^{\prime}$,

(ii) every partial isomorphism $\alpha: e L \rightarrow f L$ of $L$ can be extended to a partial isomorphism $\alpha_{L^{\prime}}: e L^{\prime} \rightarrow f L^{\prime}$ of $L^{\prime}$ such that $T_{L} \rightarrow T_{L^{\prime}}, \alpha \rightarrow \alpha_{L^{\prime}}$ embeds $T_{L}$ isomorphically in $T_{L^{\prime}}$,

(iii) every automorphism $\gamma$ of $L$ can be extended to an automorphism $\gamma_{L^{\prime}}$ of $L^{\prime}$ such that Aut $(L) \rightarrow$ Aut $\left(L^{\prime}\right), \gamma \rightarrow \gamma_{L}$ embeds Aut $(L)$ isomorphically in Aut $\left(L^{\prime}\right)$.
\end{abstract}

1980 Mathematics subject classification (Amer. Math. Soc.): primary 06 A 12; secondary $20 \mathrm{M} 10$.

We shall give a procedure for embedding any semilattice in a semilattice which has a transitive automorphism group. The particular procedure which will be given here is very likely to be relevant for the construction of inverse semigroups. The reader may consult McAlister (1974a, b, 1978) and O'Carroll (1976) for a further motivation of our embedding theorem.

We follow the terminology and the notation of Birkhoff (1967) and Howie (1976). If $L, \wedge$ is any semilattice, and $e \in L$, then the principal ideal of $L$ which is generated by $e$ will be denoted by $e L$. If $e$ and $f$ are elements of $L$ such that $\alpha: e L \rightarrow f L$ is an isomorphism, then $\alpha$ will be called a partial isomorphism of $L$. The partial isomorphisms of $L$ form an inverse subsemigroup $T_{L}$ of the symmetric

The author's research was done while he was a Visiting Professor at the University of Nebraska, supported by a Fulbright-Hays Award. 
inverse semigroup on the set $L$. This semigroup $T_{L}$ will be called the Munn semigroup of $L$ (Howie (1976), Munn (1966)). We define the relation $\mathscr{U}_{L}$ on $L$ by $\mathscr{U}_{L}=\{(e, f) \in L \times L \mid e L \cong f L\}$. The automorphism group of $L$ will be denoted by Aut $(L)$.

The following result summarizes Lemmas 1, 2 and 4 of Pastijn (preprint).

LEMMA 1. Any semilattice [lattice] $L$ can be isomorphically embedded as a filter and as a subsemilattice in a semilattice [lattice] $K$ in such a way that

(i) $L \times L \subseteq \mathscr{U}_{K}$,

(ii) every partial isomorphism $\alpha: e L \rightarrow f L$ of $L$ can be extended to a partial isomorphism $\alpha_{K}: e K \rightarrow f K$ of $K$ such that the mapping

$$
\psi: T_{L} \rightarrow T_{K}, \quad \alpha \rightarrow \alpha_{K}
$$

embeds $T_{L}$ isomorphically in $T_{K}$,

(iii) every automorphism $\gamma$ of $L$ can be extended to an automorphism $\gamma_{K}$ of $K$ such that the mapping

$$
\xi: \operatorname{Aut}(L) \rightarrow \operatorname{Aut}(K), \quad \gamma \rightarrow \gamma_{K},
$$

embeds Aut $(L)$ isomorphically in Aut $(K)$.

We now start with the embedding procedure. Let $L, \wedge$ be any semilattice. Let $L^{1}$ be the semilattice arising from $L$ by the adjunction of an identity element 1 , unless $L$ had already an identity element, in which case $L=L^{1}$. For any $\alpha \in T_{L^{1}}$, with $\alpha: e_{\alpha} L^{1} \rightarrow f_{\alpha} L^{1}$, let $L_{\alpha}$ be an isomorphic copy of $L^{1}$ which contains $f_{\alpha} L^{1}$ as a principal ideal, and $\hat{\alpha}: L^{1} \rightarrow L_{\alpha}$ an isomorphism of $L^{1}$ onto $L_{\alpha}$ which extends the partial isomorphism $\alpha$. We can always suppose that for any $\alpha, \beta \in T_{L^{1}}$, with $\alpha \neq \beta$, we have $L_{\alpha} \cap L_{\beta}=\left(f_{\alpha} \wedge f_{\beta}\right) L^{1}$. In particular, if $\alpha$ is an automorphism of $L^{1}$, then $\alpha, \beta \in T_{L^{1}}$ and $L_{\alpha}=L^{1}$. Let $L^{(1)}=\left(\bigcup_{\alpha \in T_{L^{1}}} L_{\alpha}\right) \cup\left\{1^{(1)}\right\}$. On $L^{(1)}$ we define a partial order $\leqslant$ by the following:

and

$$
x \leqslant 1^{(1)} \text { for all } x \in L^{(1)}
$$

$$
x \leqslant y, \quad x, y \in L^{(1)}, x \neq 1^{(1)} \neq y,
$$

if and only if $x, y \in L_{\alpha}$ for some $\alpha \in T_{L^{1}}$ and $x \leqslant y$ in $L_{\alpha}$.

LEMMA 2.

(i) $L^{(1)}$ is a semilattice which contains $L^{1}$ as a subsemilattice and as a principal ideal.

(ii) $L^{(1)}$ is a lattice if and only if $L^{1}$ is a lattice.

(iii) Every partial isomorphism $\alpha$ of $L$ can be extended to the partial isomorphisms $\hat{\alpha}$ and $\left(\alpha^{-1}\right)^{\wedge-1}$ of $L^{(1)}$ such that $L^{1}=\operatorname{dom} \hat{\alpha}$ and $L^{1}=\operatorname{im}\left(\alpha^{-1}\right)^{\wedge-1}$. 
(iv) Every automorphism $\gamma$ of $L$ can be extended to an automorphism $\gamma_{1}$ of $L^{(1)}$ in such a way that the mapping

$$
\xi: \operatorname{Aut}(L) \rightarrow \operatorname{Aut}\left(L^{(1)}\right), \quad \gamma \rightarrow \gamma_{1},
$$

embeds Aut $(L)$ isomorphically in $\operatorname{Aut}\left(L^{(\mathbf{1})}\right)$.

Proof. (i) Let $\alpha$ and $\beta$ be any elements of $T_{L^{1}}$, and let $x \in L_{\alpha}, y \in L_{\beta}$. If $\alpha=\beta$, then the greatest lower bound inf $(x, y)$ of $x$ and $y$ in $L^{(1)}$ equals $x \wedge y$, where the meet is to be taken in $L_{\alpha}=L_{\beta}$. In case $\alpha \neq \beta$, let $x \wedge\left(f_{\alpha} \wedge f_{\beta}\right)$ be the meet of $x$ and $f_{\alpha} \wedge f_{\beta}$ in $L_{\alpha}$, let $y \wedge\left(f_{\alpha} \wedge f_{\beta}\right)$ be the meet of $y$ and $f_{\alpha} \wedge f_{\beta}$ in $L_{\beta}$, and let

$$
\left(x \wedge\left(f_{\alpha} \wedge f_{\beta}\right)\right) \wedge\left(y \wedge\left(f_{\alpha} \wedge f_{\beta}\right)\right)
$$

be the meet of $x \wedge\left(f_{\alpha} \wedge f_{\beta}\right)$ and $y \wedge\left(f_{\alpha} \wedge f_{\beta}\right)$ in $\left(f_{\alpha} \wedge f_{\beta}\right) L^{1}$ : it is easy to see that $\left(x \wedge\left(f_{\alpha} \wedge f_{\beta}\right)\right) \wedge\left(y \wedge\left(f_{\alpha} \wedge f_{\beta}\right)\right)$ is then the greatest lower bound inf $(x, y)$ of $x$ and $y$ in $L^{(1)}$. We conclude that $L^{(1)}$, $\leqslant$ is an inf-semilattice. Since the operation 'inf' on $L^{(1)}$ extends the meet operations on the $L_{\alpha}, \alpha \in T_{L^{1}}$, there is no harm in denoting $\inf (x, y)$ by $x \wedge y$ for all $x, y \in L^{(1)}$. From this it follows in particular that $L$ is a subsemilattice of $L^{(1)}$. Furthermore, $L^{1}=1 L^{(1)}$ is a principal ideal of $L^{(1)}$.

(ii) If $L^{(1)}$ is a lattice, then every principal ideal of $L^{(1)}$ is a sublattice of $L^{(1)}$. Hence $L^{1}$ is then a sublattice of $L^{(1)}$.

Let us suppose that $L^{1}$ is a lattice, and let $x, y \in L^{(1)}$. The least upper bound $\sup (x, y)$ of $x$ and $y$ in $L^{(1)}$ equals the join $x \vee y$ of $x$ and $y$ in $L_{\alpha}$ if $x, y \in L_{\alpha}, \alpha \in T_{L^{1}}$, and $\sup (x, y)=1^{(1)}$ otherwise. $L^{(1)}$ then becomes a lattice, and the operation 'sup' on $L^{(1)}$ extends the join operations on the $L_{\alpha}, \alpha \in T_{L^{1}}$; therefore we can denote $\sup (x, y)$ by $x \vee y$ for all $x, y \in L^{(1)}$.

(iii) Trivial from our construction.

(iv) Let $\gamma$ be any automorphism of $L$. The automorphism $\gamma$ can be extended in a trivial way to an automorphism $\gamma^{\prime}$ of $L^{1}$. The automorphism $\gamma_{1}$ of $L^{(1)}$ which is defined by

and

$$
1^{(1)} \gamma_{1}=1^{(1)}
$$

$$
x \gamma_{1}=x \hat{\alpha}^{-1}\left(\alpha \gamma^{\prime}\right)^{\wedge} \quad \text { if } x \in L_{\alpha}, \quad \alpha \in T_{L^{1}},
$$

extends $\gamma$ and the mapping $\xi$ : Aut $(L) \rightarrow \operatorname{Aut}\left(L^{(1)}\right), \gamma \rightarrow \gamma_{1}$, is injective. Furthermore, if $\gamma, \delta \in \operatorname{Aut}(L), x \in L_{\alpha}, \alpha \in T_{L^{1}}$, then

$$
\begin{aligned}
x \gamma_{1} \delta_{1} & =\left(x \hat{\alpha}^{-1}\left(\alpha \gamma^{\prime}\right)^{\wedge}\right) \delta_{1} \\
& =x \hat{\alpha}^{-1}\left(\alpha \gamma^{\prime}\right)^{\wedge}\left(\alpha \gamma^{\prime}\right)^{\wedge-1}\left(\alpha \gamma^{\prime} \delta^{\prime}\right)^{\wedge} \\
& =x \hat{\alpha}^{-1}\left(\alpha(\gamma \delta)^{\prime}\right)^{\wedge} \\
& =x(\gamma \delta)_{1} .
\end{aligned}
$$

Thus $\xi$ is an isomorphism of $\operatorname{Aut}(L)$ into $\operatorname{Aut}\left(L^{(1)}\right)$. 
In McAlister (1974b), Theorem 4.2 it was proved that every semilattice $L$ can be embedded as an ideal in a partially ordered set $\mathscr{X}$ in such a way that every partial isomorphism of $L$ can be extended to an automorphism of $\mathscr{X}$. Slight refinements for this result may be obtained from McAlister (1976), Section 3. Corollary 4.4 of McAlister (1978) states that in Theorem 4.2 of McAlister (1974b) $\mathscr{X}$ can be chosen to be a semilattice. Using quite different techniques, this result was also obtained in Section 2 of Meakin and Pastijn (preprint). We now present a theorem which is an application of our Lemma 2, and which is a refinement for the results mentioned here. It should be remarked that the following theorem could also be obtained using Lemma 1.2 of O'Carroll (1976), together with the results of McAlister (1974a, b). Our construction, however, has the advantage that the proofs are purely semilattice-theoretic and independent of any knowledge about the construction of $E$-unitary inverse semigroups.

THEOREM 3. Every semilattice [lattice] $L$ can be embedded isomorphically as an ideal in a semilattice [lattice] $L^{\prime}$ in such a way that

(i) every partial isomorphism $\alpha$ of $L^{\prime}$ can be extended to an automorphism $\bar{\alpha}$ of $L^{\prime}$,

(ii) every automorphism $\gamma$ of $L$ can be extended to an automorphism $\gamma^{\prime}$ of $L^{\prime}$ in such a way that the mapping

$$
\xi: \operatorname{Aut}(L) \rightarrow \operatorname{Aut}\left(L^{\prime}\right), \quad \gamma \rightarrow \gamma^{\prime},
$$

embeds Aut $(L)$ isomorphically in Aut $\left(L^{\prime}\right)$.

Proof. Let us consider the sequence of semilattices

$$
L=L^{(0)}, L^{(1)}, L^{(2)}, \ldots, L^{(j)}, L^{(j+1)}, \ldots
$$

where for each $j, L^{(j+1)}$ is constructed from $L^{(j)}$ in the way described before Lemma 2. It is then clear from Lemma 2(i) that $L^{\prime}=\bigcup_{j=0}^{\infty} L^{(j)}$ is a semilattice which contains $L$ as an ideal. From Lemma 2(ii) it follows that $L^{\prime}$ is a lattice if $L$ is a lattice. Let $x, y \in L^{\prime}$, and $\alpha: x L^{\prime} \rightarrow y L^{\prime}$ a partial isomorphism of $L^{\prime}$. There exists a $j$ such that $x, y \in L^{(j)}$, and then $x L^{\prime}=x L^{(j)}, y L^{\prime}=y L^{(j)}$ since $L^{(j)}$ must be an ideal of $L^{\prime}$. Hence $\alpha$ is a partial isomorphism of $L^{(j)}$. From Lemma 2(iii) it now follows that we can consider a sequence

$$
\alpha=\alpha^{(0)}, \alpha^{(1)}, \ldots, \alpha^{(k)}, \alpha^{(k+1)}, \ldots
$$

in which for every $k \alpha^{(k+1)}$ is a partial isomorphism of $L^{(j+k+1)}$ which extends the partial isomorphism $\alpha^{(k)}$ of $L^{(j+k)}$ such that

and

$$
L^{(j+k-1)}=\operatorname{dom} \alpha^{(k)} \quad \text { if } k \text { is odd }
$$

$$
L^{(j+k-1)}=\operatorname{im} \alpha^{(k)} \quad \text { if } k \text { is even, } k \geqslant 2 .
$$

It follows that $\bigcup_{k=0}^{\infty} \alpha^{(k)}=\bar{\alpha}$ is an automorphism of $L^{\prime}$ which extends the partial isomorphism $\alpha$. Thus condition (i) is satisfied.

(ii) follows from Lemma 2(iv). 
Note that every partial isomorphism of $L$ is also a partial isomorphism of $L^{\prime}$. Therefore Theorem 3(i) guarantees that every partial isomorphism of $L$ can be extended to an automorphism of $L^{\prime}$.

Recall that a convex subsemilattice [sublattice] of a semilattice [lattice] is the intersection of an ideal and a filter.

THEOREM 4. Every semilattice [lattice] $L$ can be isomorphically embedded as a convex subsemilattice [sublattice] in a semilattice [lattice] $L^{\prime}$ which has a transitive automorphism group in such a way that

(i) every partial isomorphism $\alpha$ of $L$ can be extended to an automorphism $\bar{\alpha}$ of $L^{\prime}$,

(ii) every partial isomorphism $\alpha: e L \rightarrow f L$ of $L$ can be extended to a partial isomorphism $\alpha_{L^{\prime}}: e L^{\prime} \rightarrow f L^{\prime}$ of $L^{\prime}$ such that the mapping

$$
\psi: T_{L} \rightarrow T_{L^{\prime}}, \quad \alpha \rightarrow \alpha_{L^{\prime}},
$$

embeds $T_{L}$ isomorphically in $T_{L^{\prime}}$,

(iii) every automorphism $\gamma$ of $L$ can be extended to an automorphism $\gamma_{L^{\prime}}$ of $L^{\prime}$ such that the mapping

$$
\xi: \operatorname{Aut}(L) \rightarrow \operatorname{Aut}\left(L^{\prime}\right), \quad \gamma \rightarrow \gamma_{L^{\prime}}
$$

embeds Aut $(L)$ isomorphically in Aut $\left(L^{\prime}\right)$.

Proor. Let us consider the sequence of semilattices

$$
L=L_{0}, K_{0}, L_{1}, K_{1}, \ldots, L_{j}, K_{j}, L_{j+1}, K_{j+1}, \ldots,
$$

where for each $j K_{j}$ is constructed from $L_{j}$ in the way prescribed by Lemma 1, and where for each $j L_{j+1}$ is constructed from $K_{j}$ in the same way as $L^{(1)}$ was constructed from $L$ (see before Lemma 2). It is then clear from Lemma 1 and Lemma 2(i) that $L^{\prime}=\bigcup_{j=0}^{\infty} L_{j}=\bigcup_{j=0}^{\infty} K_{j}$ is a semilattice which contains $L$ as a convex subsemilattice. From Lemma 1 and Lemma 2(i) it follows that $L^{\prime}$ is a lattice if $L$ is a lattice. Let $\alpha^{(j)}$ be a partial isomorphism of $L_{j}$ for some $j \in \mathbf{N}$. From Lemma 1(ii) and from Lemma 2(iii) it now follows that we can consider a sequence

$$
\alpha^{(j)}, \alpha_{K_{j}}^{(j)}, \alpha^{(j+1)}, \alpha_{K_{j+1}}^{(j+1)}, \ldots,
$$

where for any $k \geqslant j, \alpha_{K_{k}}^{(k)}$ is a partial isomorphism of $K_{k}$ which extends the partial isomorphism $\alpha^{(k)}$ of $L^{(k)}$, and $\alpha^{(k+1)}$ a partial isomorphism of $L^{(k+1)}$ which extends the partial isomorphism $\alpha_{K_{k}}^{(k)}$ of $K_{k}$ such that

and

$$
K_{k}^{1}=\operatorname{dom} \alpha^{(k+1)} \quad \text { if } k \text { is odd }
$$

$$
K_{k}^{1}=\operatorname{im} \alpha^{(k+1)} \text { if } k \text { is even. }
$$


It follows that $\bigcup_{k=j}^{\infty} \alpha^{(k)}$ is an automorphism of $L^{\prime}$ which extends $\alpha^{(j)}$. In particular, every partial isomorphism $\alpha$ of $L$ can be extended to an automorphism $\tilde{\alpha}$ of $L$, and so (i) is satisfied.

Let us consider any two elements $x, y \in L^{\prime}$. There exists a $j$ such that $x, y \in L_{j-1}$. By Lemma 1 (i) we know that $(x, y) \in \mathscr{U}_{K_{j-1}}$, and so there exists a partial isomorphism $\alpha_{K_{j-1}}$ of $K_{j-1}$ and $L_{j}$ which maps $x$ onto $y$. Since $\alpha_{K_{j-1}}$ can be extended to an automorphism of $L^{\prime}$ we can now conclude that Aut $\left(L^{\prime}\right)$ acts transitively on $L^{\prime}$.

We know that for any $j K_{j}$ is an ideal of $L_{j+1}$; consequently $T_{K}$, is a subsemigroup of $T_{L_{i+1}}$. From this fact, and from Lemma 1(ii) we conclude that condition (ii) is satisfied.

From Lemma 1(iii) and from Lemma 2(iv) it follows that condition (iii) is satisfied. This concludes the proof of the theorem.

Note that a lattice $L^{\prime}$ which has a transitive automorphism group must a fortiori be uniform, and $T_{L^{\prime}}$ must be bisimple. That any semilattice can be isomorphically embedded as a subsemilattice in a uniform semilattice also follows from Reilly (1965).

From Pastijn (preprint) and our present construction it actually follows that in Theorem $4 L$ is embedded as an open interval in $L^{\prime}$. If $L$ has an identity and a zero, then $L$ is of course embedded as a closed interval in $L^{\prime}$.

\section{References}

G. Birkhoff (1967), Lattice theory (Amer. Math. Soc. Colloq. Publ., New York).

J. M. Howie (1976), An introduction to semigroup theory (Academic Press, London).

D. B. McAlister (1974a). 'Groups, semilattices and inverse semigroups', Trans. Amer. Math. Soc., 192, 227-244.

D. B. McAlister (1974b), 'Groups, semilattices and inverse semigroups II', Trans. Amer. Math. Soc. 196, 351-369.

D. B. McAlister (1976), 'Some covering and embedding theorems for inverse semigroups', J. Austral. Math. Soc. 22, 188-211.

D. B. McAlister (1978), 'E-unitary inverse semigroups over semilattices', Glasgow Math. J. 19, 1-12.

J. Meakin and F. Pastijn, 'The structure of pseudo-semilattices', preprint.

W. D. Munn (1966), 'Uniform semilattices and bisimple inverse semigroups', Quart. J. Math. Oxford 17, 151-159.

L. O'Carroll (1976), 'Embedding theorems for proper inverse semigroups', J. Algebra 42, 26-40.

F. Pastijn, 'Uniform lattices', preprint.

N. R. Reilly (1965), 'Embedding inverse semigroups in bisimple inverse semigroups', Quart J. Math. Oxford 16, 183-187.

University of Nebraska-Lincoln

Lincoln, NE 68588

U.S.A.
Rijksuniversiteit Gent B-9000 Gent

Belgium 